\title{
Insegurança alimentar e nutricional de famílias com crianças menores de cinco anos da Região Metropolitana de João Pessoa, Paraíba, Brasil
}

\author{
Food and nutrition insecurity among families with children under \\ five years of age in the Metropolitan Region of João Pessoa, Paraíba, \\ Brazil
}

Dixis Figueroa Pedraza (http://orcid.org/0000-0002-5394-828X) ${ }^{1}$

${ }^{1}$ Universidade Estadual da Paraíba. Av. das Baraúnas 351, Bairro Bodocongó. 58109-753 Campina Grande PB Brasil. dixisfigueroa@gmail.com

\begin{abstract}
The scope of this article is to evaluate the food and nutrition insecurity of families with children under five years of age residing in $\mathrm{mu}$ nicipalities of the Metropolitan Region of João Pessoa; its association with biological characteristics; the health status of the children and the family socioeconomic context. It is a cross-sectional study with families attended in the Family Health Strategy. Food and nutritional security were evaluated by the Brazilian Food Insecurity Scale. The association of moderate/severe food and nutrition insecurity with socioeconomic and child characteristics was studied using Multivariate Logistic Regression. The prevalence of food and nutrition insecurity was found in $63.9 \%$ of families, the moderate/severe forms being predominant. Families whose children had diarrhea in the last month, where mothers indicated that they were unable to read/write/do accounts, among beneficiaries of the Bolsa Familia Program and with $<2$ minimum wages per capita family income, presented higher prevalence of moderate/severe food and nutrition insecurity. The high vulnerability of the families observed, especially the beneficiaries of Bolsa Familia, is reflected in more severe degrees of food and nutrition insecurity associated with socioeconomic factors.
\end{abstract}

Key words Food and Nutrition Security, Social Vulnerability, Socioeconomic Factors
Resumo O objetivo deste artigo é avaliar a insegurança alimentar e nutricional de famílias com crianças menores de cinco anos residentes em municípios da Região Metropolitana de João Pessoa; sua associação com características biológicas; da situação de saúde das crianças e do contexto socioeconômico familiar. Estudo transversal com famílias atendidas na Estratégia Saúde da Família. A segurança alimentar e nutricional foi avaliada pela Escala Brasileira de Insegurança Alimentar. A associação entre a insegurança alimentar e nutricional moderada/grave e as características de interesse foi avaliada por meio de Regressão Logística Multivariada. A prevalência de insegurança alimentar e nutricional foi encontrada em 63,9\% das famílias, predominando as formas moderada/ grave. As famílias cujas crianças tiveram diarreia no último mês, naquelas cujas mães indicaram não saber ler/escrever/fazer contas, entre beneficiárias do Programa Bolsa Família e com renda familiar per capita <2 salários minimos apresentaram maiores prevalências de insegurança alimentar e nutricional moderada/grave. A alta vulnerabilidade das famílias observadas, sobretudo as beneficiárias do Bolsa Família, reflete-se em graus mais severos de insegurança alimentar e nutricional, associados a fatores socioeconômicos. Palavras-chave Segurança Alimentar e Nutricional, Vulnerabilidade Social, Fatores Socioeconômicos 


\section{Introdução}

A fome lidera a lista dos dez maiores riscos à saúde e é considerada o maior problema solucionável do mundo ${ }^{1}$. Embora a segurança alimentar e nutricional constitua um direito fundamental, depois de um prolongado descenso, o número de pessoas com fome no mundo aumentou significativamente, atingindo 815 milhões de pessoas em $2016^{2}$.

Por meio da Escala de Experiência de Insegurança Alimentar, a Organização das Nações Unidas para Agricultura e Alimentação estima que uma a cada 10 pessoas $(9,3 \%$ ou 689 milhões de pessoas), de aproximadamente 150 países, estava em insegurança alimentar e nutricional grave ou fome, segundo dados de 2014, 2015 e 2016. Na América Latina, a insegurança alimentar e nutricional grave aumentou de $4,7 \%$ para $6,4 \%$ durante o triênio ${ }^{2}$. A situação no Brasil não é diferente; estima-se que 17,5\% das famílias com crianças menores de cinco anos residem em domicílios com insegurança alimentar e nutricional moderada/grave, com prevalências concentradas nas regiões Norte e Nordeste (30,7\%), nas classes econômicas de pior situação (34\%) e em beneficiários de programas de transferência de renda $(36,5 \%)^{3}$. Medida por meio da percepção e experiência com a fome, esses indicadores referem-se à insegurança alimentar e nutricional definida como o acesso limitado aos alimentos devido à falta de dinheiro ou outros recursos ${ }^{2}$. Para a população brasileira, a segurança alimentar e nutricional representa "a realização do direito de todos ao acesso regular e permanente a alimentos de qualidade, em quantidade suficiente, sem comprometer o acesso a outras necessidades essenciais, tendo como base práticas alimentares promotoras de saúde, que respeitem a diversidade cultural e que sejam social, econômica e ambientalmente sustentáveis"4.

A determinação social da insegurança alimentar e nutricional no Brasil implica na necessidade de dados desagregados sobre grupos específicos, sugerindo-se maiores prevalências do problema nos casos de populações em iniquidade social, no contexto escolar e entre usuários de serviços de saúde/favorecidos pelo Programa Bolsa Família (PBF), em relação à população geral $^{5}$. Bezerra et al. ${ }^{5}$, em revisão sistemática da literatura, apontaram a renda familiar, o número de indivíduos no domicílio e o tipo de moradia como principais fatores de exposição na deter- minação da insegurança alimentar e nutricional, mas apenas um dos estudos incluídos com amostras de populações procedentes de serviços de saúde/beneficiários do PBF conduziu análises ajustadas de associação ${ }^{5}$.

No município de Campina Grande-PB foram conduzidos dois estudos envolvendo famílias com crianças menores de cinco anos em dois contextos diferentes: creches $^{6}$ e residentes em área de vulnerabilidade social ${ }^{7}$. Os resultados desses estudos indicaram altas prevalências de insegurança alimentar e nutricional, de $64,2 \%$ no contexto escolar ${ }^{6}$ e de $96,1 \%$ no grupo em iniquidade social ${ }^{7}$. Ainda, considerando os graus mais graves de insegurança alimentar e nutricional (moderada/grave), as prevalências foram $31,6 \%{ }^{6}$ e $66,6 \%{ }^{7}$. No contexto das creches, maiores prevalências de insegurança alimentar e nutricional moderada/grave estiveram presentes entre famílias de domicílios sem tratamento da água para beber, com outro tipo de sanitário que não individual com descarga, nas mais numerosas e sem posse de geladeira ${ }^{6}$. Para as famílias de vulnerabilidade social, situação similar foi constatada nos casos de crianças que tiveram perda de peso nos últimos 15 dias e de domicílios com destino de lixo não coletado? .

O estudo atual continua na linha de raciocínio precedente, selecionando-se famílias atendidas por equipes de saúde da família e delimitando-se o benefício do PBF. A importância desse cenário relaciona-se às possibilidades que a Estratégia Saúde da Família (ESF) pode proporcionar no cuidado integral e na promoção da saúde, sobretudo com base nas desigualdades sociais. A ESF pode ter papel essencial na garantia das condicionalidades de saúde e nutrição entre beneficiários do PBF e na adoção de outros tipos de intervenções articuladas a este Programa, como ações de promoção da alimentação saudável e a suplementação de alimentos ${ }^{8,9}$. Ainda, a identificação da vulnerabilidade à insegurança alimentar e nutricional é essencial ao planejamento de políticas públicas, destacando-se divergência de achados em relação aos efeitos do $\mathrm{PBF}^{5,6}$.

Este estudo teve por objetivo avaliar a situação de insegurança alimentar e nutricional de famílias com crianças menores de cinco anos residentes em municípios da Região Metropolitana de João Pessoa; sua associação com características biológicas; da situação de saúde das crianças e do contexto socioeconômico familiar. 


\section{Metodologia}

Estudo de delineamento transversal conduzido no contexto da ESF em dois municípios do estado da Paraíba, selecionados por conveniência. A escolha dos municípios considerou suas semelhanças quanto à posição geográfica (localização na região metropolitana da capital do Estado com acesso à rede de serviços nela disponibilizados), grau de urbanização (de quase 100\%), indicadores demográfico-sociais (índice de desenvolvimento humano de nível médio) e tradição na organização de serviços primários de saúde (cobertura populacional da ESF de quase 100\%). Um dos municípios tem população de 57.944 habitantes, dos quais 4.596 são crianças menores de cinco anos, e possui sistema de saúde composto por 19 equipes da ESF. O outro município tem população de 99.716 habitantes, dos quais 7.862 são crianças menores de cinco anos, e possui sistema de saúde composto por 28 equipes da ESF.

Crianças menores de cinco anos constituíram a população de estudo. Tomando-se por base para o cálculo amostral um nível de $95 \%$ de confiança; poder do teste de $80 \%$; relação entre expostos e não expostos à intervenção (benefício do PBF) de 1:1; prevalência de desfechos de no mínimo $25 \%$; $10 \%$ para compensar eventuais perdas; $15 \%$ para controle de fatores de confusão e $60 \%$ para efeito de delineamento, estimou-se a necessidade de estudar 324 sujeitos.

A amostra foi selecionada por conglomerados em dois estágios. No primeiro, foram sorteadas aleatoriamente as equipes de saúde; no segundo estágio, um número fixo de crianças. Em cada município foram selecionadas nove equipes da ESF que representaram, no mínimo, $1 / 3$ do total municipal. Cada equipe contribuiu, em média, com 18 crianças para o total da amostra que obedeceu a dois critérios: i) uma amostra intencional, composta por todos os atendimentos nas consultas de puericultura no dia da coleta de dados, em um dia típico de trabalho; ii) uma amostra de crianças encaminhadas aos serviços de saúde pelos agentes comunitários de saúde, conforme rotina de trabalho, das quais selecionaram-se aleatoriamente a quantidade que permitisse completar o total previsto para cada equipe de saúde.

A coleta foi realizada nas unidades de saúde entre julho e dezembro de 2014. A equipe de campo do estudo foi formada por profissionais e estudantes da área de saúde, com experiência prévia em trabalho de campo, o qual foi supervisionado por profissional capacitado. $\mathrm{O}$ controle de qualidade do estudo incluiu o uso de um Manual de
Instruçães e o treinamento e padronização dos entrevistadores. Além disso, foi realizado um estudo piloto no município de Campina Grande, contemplando o teste dos instrumentos e entrevistadores em 32 pessoas ( $10 \%$ da amostra) que se enquadravam nos critérios de inclusão da população pretendida para o estudo.

Para avaliar a situação de segurança alimentar das famílias, foi aplicada às mães, acompanhado de um questionário socioeconômico, a Escala Brasileira de Insegurança Alimentar (EBIA), composta por 15 perguntas, com respostas positivas e negativas relativas à percepção da entrevistada sobre a situação alimentar vivida pela família nos últimos três meses anteriores à entrevista. Para as respostas positivas, foi atribuído o valor 1 (um) e, para as negativas, o valor 0 (zero). Quando a entrevistada respondeu negativamente a todas as questões da escala, a família foi classificada em situação de segurança alimentar; até 5 perguntas positivas, insegurança alimentar leve; de 6 a 10 respostas positivas, insegurança alimentar moderada, e de 11 a 15 respostas positivas, insegurança alimentar grave ${ }^{10}$.

A insegurança alimentar e nutricional foi a variável dependente de estudo. Para análises, o desfecho foi dicotomizado em segurança alimentar e nutricional/insegurança alimentar e nutricional leve (situação de referência), e insegurança alimentar e nutricional moderada/grave. Além disso, analisou-se o comportamento segundo as categorias extremas de segurança alimentar e nutricional (segurança alimentar e nutricional vs. insegurança alimentar e nutricional grave).

Como variáveis independentes foram consideradas características biológicas e da situação de saúde das crianças, e do contexto socioeconômico familiar. As características biológicas e da situação de saúde das crianças incluíram: sexo, idade, peso ao nascer, saúde da criança, internação nos últimos 12 meses, pneumonia nos últimos 6 meses, diarreia no último mês. As variáveis relacionadas à situação de saúde das crianças foram referidas pelas mães. Peso ao nascer $<2.500 \mathrm{~g}$ definiu o baixo peso da criança ao nascimento. As características do contexto socioeconômico familiar avaliadas foram: escolaridade materna, trabalho materno fora de casa, número de pessoas no domicílio, esgotamento sanitário do domicílio, benefício do PBF, renda familiar per capita.

$\mathrm{Na}$ primeira etapa das análises, as associações bivariadas entre o desfecho e as variáveis independentes foram investigadas utilizando o Teste qui-quadrado, considerando o nível de significância de 5\%. Na análise de Regressão Logística 
Multivariada, foram incluídas no modelo todas as variáveis independentes que estiveram estatisticamente associadas ao evento na análise bivariada $(\mathrm{p}<0,25)$. Permaneceram no modelo final somente as variáveis que apresentaram valores de $\mathrm{p}<0,05$. Também foram calculados as razões de prevalência (RP) e os respectivos intervalos de confiança (IC95\%) para as categorias extremas de segurança alimentar (segurança alimentar e nutricional vs. insegurança alimentar e nutricional grave). As análises foram realizadas com o auxílio do programa estatístico R.

O projeto foi aprovado pelo Comitê de Ética em Pesquisa da Universidade Estadual da Paraíba. A relevância e os objetivos da pesquisa foram devidamente explicados aos participantes, procedimento após o qual foi assinado o Termo de Consentimento Livre e Esclarecido, condição necessária para a participação no estudo. Foi garantida a todos os sujeitos envolvidos a liberdade de não participar da pesquisa ou dela desistir, a privacidade da imagem e a confidencialidade das informações.

\section{Resultados}

Considerando as prevalências, verifica-se que $63,9 \%$ das famílias estavam com algum grau de insegurança alimentar e nutricional, principalmente nas formas moderada/grave $(44,6 \%)$. A insegurança alimentar e nutricional grave atingiu 15,0\% dos domicílios (Figura 1).
Dentre os problemas de saúde analisados, a ocorrência de diarreia no último mês anterior à entrevista foi o de maior frequência (22,5\%). Apenas 23,4\% das mães trabalhavam fora de casa e 19,6\% dos domicílios tinham rede pública de esgoto domiciliar. Do total de famílias, $40,2 \%$ não recebiam benefício do PBF e 72,2\% tinham renda familiar per capita inferior a dois salários mínimos (Tabela 1).

Maiores prevalências de insegurança alimentar e nutricional moderada/grave foram encontradas nas famílias cujas crianças apresentaram diarreia no último mês que antecedeu a coleta dos dados $(\mathrm{p}=0,0456)$, naquelas cujas mães indicaram não saber ler, escrever e fazer contas $(\mathrm{p}=0,0013)$, entre beneficiárias do PBF $(\mathrm{p}<0,0001)$ e com renda familiar per capita $<2$ salários mínimos $(\mathrm{p}<0,0001)$ (Tabela 1$)$.

No modelo ajustado, todas as variáveis anteriores mantiveram significância estatística, sendo a renda familiar per capita $<2$ salários mínimos a que apresentou maior RP de insegurança alimentar e nutricional moderada/grave, duas vezes maior (IC95\%=1,35; 2,94). A exclusão do PBF representou um potencial fator de risco para os maiores graus de insegurança alimentar (Tabela 2).

Para as análises das categorias extremas de segurança alimentar e nutricional, mostradas na Tabela 3, com exceção da diarreia no último mês, as associações foram similares. Constataram-se maiores prevalências de insegurança alimentar e nutricional grave em contextos socioeconômicos desfavoráveis: pior escolaridade materna, menor renda familiar per capita e benefício do PBF.

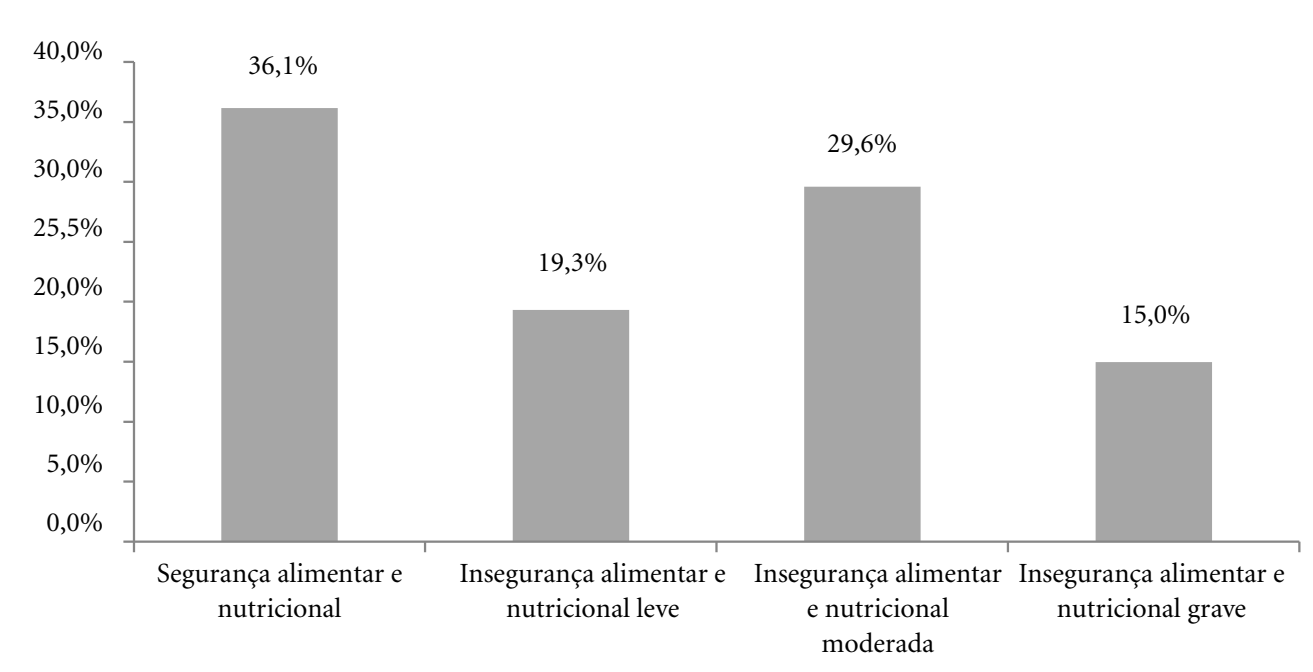

Figura 1. Prevalências de (in)segurança alimentar e nutricional em famílias com crianças menores de cinco anos residentes em municípios da Região Metropolitana de João Pessoa-PB, 2014. 
Tabela 1. Situação de segurança alimentar e nutricional, de acordo com características biológicas e da situação de saúde das crianças, e do contexto socioeconômico familiar. Municípios da Região Metropolitana de João PessoaPB, 2014.

\begin{tabular}{|c|c|c|c|c|c|c|c|}
\hline \multirow[t]{2}{*}{ Variáveis } & \multicolumn{2}{|c|}{ Total } & \multicolumn{2}{|c|}{$\begin{array}{l}\text { Segurança alimentar } \\
\text { e nutricional/ } \\
\text { insegurança alimentar } \\
\text { e nutricional leve }\end{array}$} & \multicolumn{2}{|c|}{$\begin{array}{c}\text { Insegurança } \\
\text { alimentar e } \\
\text { nutricional } \\
\text { moderada/grave }\end{array}$} & \multirow[t]{2}{*}{$\begin{array}{l}\text { Valor } \\
\text { de p }\end{array}$} \\
\hline & $\mathrm{n}$ & $\%$ & $\mathbf{n}$ & $\%$ & $\mathbf{n}$ & $\%$ & \\
\hline \multicolumn{8}{|l|}{ Características da Criança } \\
\hline Sexo & & & & & & & 0,0895 \\
\hline Masculino & 164 & 51,1 & 99 & 60,4 & 65 & 39,6 & \\
\hline Feminino & 157 & 48,9 & 79 & 50,3 & 78 & 49,7 & \\
\hline Idade (meses) & & & & & & & 0,3161 \\
\hline$\geq 25$ e $<60$ & 80 & 25,0 & 40 & 50,0 & 40 & 50,0 & \\
\hline$<25$ & 241 & 75,0 & 138 & 57,3 & 103 & 42,7 & \\
\hline Peso ao nascer & & & & & & & 0,5552 \\
\hline Normo peso $(\geq 2.500 \mathrm{~g})$ & 248 & 92,2 & 141 & 56,8 & 107 & 43,2 & \\
\hline Baixo peso & 21 & 7,8 & 10 & 47,6 & 11 & 52,4 & \\
\hline Saúde & & & & & & & 0,5844 \\
\hline Muito boa/boa & 270 & 84,1 & 152 & 56,3 & 118 & 43,7 & \\
\hline Regular/ruim & 51 & 15,9 & 26 & 51,0 & 25 & 49,0 & \\
\hline Internação nos últimos 12 meses & & & & & & & 0,6169 \\
\hline Não & 276 & 86,0 & 151 & 54,7 & 125 & 45,3 & \\
\hline $\operatorname{Sim}$ & 45 & 14,0 & 27 & 60,0 & 18 & 40,0 & \\
\hline Pneumonia nos últimos 6 meses & & & & & & & 0,3326 \\
\hline Não & 302 & 94,0 & 170 & 56,3 & 132 & 43,7 & \\
\hline Sim & 19 & 6,0 & 8 & 42,1 & 11 & 57,9 & \\
\hline Diarreia no último mês & & & & & & & 0,0456 \\
\hline Não & 249 & 77,5 & 146 & 58,6 & 103 & 41,4 & \\
\hline $\operatorname{Sim}$ & 72 & 22,5 & 32 & 44,4 & 40 & 55,6 & \\
\hline \multicolumn{8}{|l|}{ Contexto Socioeconômico } \\
\hline Escolaridade materna & & & & & & & 0,0013 \\
\hline Sabe ler, escrever e fazer contas & 254 & 79,1 & 153 & 60,2 & 101 & 39,8 & \\
\hline Não sabe ler, escrever e fazer contas & 67 & 20,9 & 25 & 37,3 & 42 & 62,7 & \\
\hline Trabalho materno fora de casa & & & & & & & 0,2993 \\
\hline Sim & 75 & 23,4 & 46 & 61,3 & 29 & 38,7 & \\
\hline Não & 246 & 76,6 & 132 & 53,7 & 114 & 46,3 & \\
\hline Esgotamento sanitário do domicílio & & & & & & & 0,9022 \\
\hline Rede pública & 63 & 19,6 & 34 & 54,0 & 29 & 46,0 & \\
\hline Outros & 258 & 80,4 & 144 & 55,8 & 114 & 44,2 & \\
\hline Número de pessoas no domicílio & & & & & & & 0,4337 \\
\hline$<4$ & 106 & 33,0 & 55 & 51,9 & 51 & 48,1 & \\
\hline$\geq 4$ & 215 & 67,0 & 123 & 57,2 & 92 & 42,8 & \\
\hline Benefício do Programa Bolsa Família & & & & & & & $<0,0001$ \\
\hline Sim & 192 & 59,8 & 88 & 45,8 & 104 & 54,2 & \\
\hline Não & 129 & 40,2 & 90 & 69,8 & 39 & 30,2 & \\
\hline Renda familiar per capita (salário mínimo) & & & & & & & $<0,0001$ \\
\hline$\geq 2$ & 88 & 27,8 & 70 & 79,5 & 18 & 20,5 & \\
\hline$<2$ & 228 & 72,2 & 105 & 46,1 & 123 & 53,9 & \\
\hline
\end{tabular}


Tabela 2. Razão de Prevalência e Intervalo de Confiança de 95\% entre as características das crianças (biológicas e da situação de saúde) e do contexto socioeconômico familiar com a insegurança alimentar e nutricional moderada/grave. Modelo de Regressão Logística Multivariada. Municípios da Região Metropolitana de João Pessoa-PB, 2014.

\begin{tabular}{lcc}
\hline \multicolumn{1}{c}{ Variáveis } & \multicolumn{1}{c}{ Insegurança alimentar e nutricional moderada/grave } \\
\cline { 2 - 3 } & RP & IC95\% \\
\hline Características da Criança & & - \\
Diarreia no último mês & 1,00 & 1,$03 ; 1,66$ \\
$\quad$ Não & 1,31 & \\
$\quad$ Sim & & - \\
Contexto Socioeconômico & & 1,$16 ; 1,86$ \\
Escolaridade materna & 1,00 & - \\
$\quad$ Sabe ler, escrever e fazer contas & 1,47 & \\
$\quad$ Não sabe ler, escrever e fazer contas & & 0,$55 ; 0,95$ \\
Benefício do Programa Bolsa Família & 1,00 & \\
$\quad$ Sim & 0,73 & 1,$35 ; 2,94$ \\
$\quad$ Não & & \\
Renda familiar per capita (salário mínimo) & 1,00 & \\
$\quad \geq 2$ & 2,00 & \\
$\quad<2$ & & \\
\hline RP: Razão de Prevalência, IC 95\%: Intervalo de Confiança de 95\%. & & \\
\end{tabular}

Tabela 3. Razão de Prevalência e Intervalo de Confiança de 95\% entre as características das crianças (biológicas e da situação de saúde) e do contexto socioeconômico familiar considerando as categorias extremas de segurança alimentar e nutricional (segurança alimentar e nutricional $v s$. insegurança alimentar e nutricional grave). Municípios da Região Metropolitana de João Pessoa-PB, 2014.

\begin{tabular}{|c|c|c|c|c|}
\hline \multirow[t]{2}{*}{ Variáveis } & \multicolumn{2}{|c|}{$\begin{array}{c}\text { Segurança } \\
\text { alimentar e } \\
\text { nutricional }\end{array}$} & \multicolumn{2}{|c|}{$\begin{array}{c}\text { Insegurança } \\
\text { alimentar e } \\
\text { nutricional grave }\end{array}$} \\
\hline & RP & IC95\% & $\mathbf{R P}$ & IC95\% \\
\hline \multicolumn{5}{|l|}{ Características da Criança } \\
\hline \multicolumn{5}{|l|}{ Diarreia no último mês } \\
\hline Não/sim & 0,86 & 0,$66 ; 1,12$ & & \\
\hline Sim/não & & & 1,16 & 0,$89 ; 1,50$ \\
\hline \multicolumn{5}{|l|}{ Contexto Socioeconômico } \\
\hline \multicolumn{5}{|l|}{ Escolaridade materna } \\
\hline $\begin{array}{l}\text { Sabe ler, escrever e fazer contas/Não sabe ler, escrever e } \\
\text { fazer contas }\end{array}$ & 0,67 & 0,$47 ; 0,95$ & & \\
\hline $\begin{array}{l}\text { Não sabe ler, escrever e fazer contas/Sabe ler, escrever e } \\
\text { fazer contas }\end{array}$ & & & 1,50 & 1,$06 ; 2,13$ \\
\hline \multicolumn{5}{|l|}{ Benefício do Programa Bolsa Família } \\
\hline Sim/Não & 1,37 & 1,$09 ; 1,71$ & & \\
\hline Não/Sim & & & 0,73 & 0,$58 ; 0,98$ \\
\hline \multicolumn{5}{|l|}{ Renda familiar per capita (salário mínimo) } \\
\hline$\geq 2 /<2$ & 0,52 & 0,$42 ; 0,65$ & & \\
\hline$<2 / \geq 2$ & & & 1,91 & 1,$54 ; 2,36$ \\
\hline
\end{tabular}

RP: Razão de Prevalência, IC95\%: Intervalo de Confiança de 95\%. 
A prevalência de insegurança alimentar e nutricional $(63,9 \%)$ foi maior do que a encontrada para as famílias brasileiras $(22,6 \%)$, da região Nordeste $(38,1 \%)$ e do estado da Paraíba (36,5\%), segundo a Pesquisa Nacional por Amostra de Domicílios (PNAD) de $2013^{11}$. Prevalências similares às deste estudo foram constatadas entre famílias do norte de Alagoas $(63,6 \%)^{12}$, com crianças assistidas em creches de Campina Grande-PB $(64,2 \%)^{6}$, em comunidades quilombolas $(64,9 \%)^{13}$ e cobertas pela ESF de Teresina-PI $(65,0 \%)^{14}$. Entretanto, destaca-se, igualmente, que o valor não ultrapassa a prevalência média ponderada $(76,6 \%)$ obtida em revisão sistemática que considerou os resultados de seis estudos com amostras obtidas de serviços de saúde/beneficiários do $\mathrm{PBF}^{5}$. Dessa forma, reforça-se a vulnerabilidade à insegurança alimentar e nutricional nas famílias cobertas pela ESF, mostrando-se que a população estudada apresenta insegurança alimentar e nutricional associada aos baixos níveis de renda, aquém dos encontrados nos níveis nacionais, regionais e estaduais ${ }^{3,11}$.

Outro fato a ser destacado foi a alta prevalência de insegurança alimentar e nutricional grave $(15,0 \%)$, superior à encontrada para as famílias brasileiras $(3,2 \%)$, nordestinas $(5,6 \%)$ e paraibanas $(3,3 \%)^{11}$. Entretanto, assemelha-se às taxas encontradas em municípios do norte de Alagoas $(14,2 \%)^{12}$, no contexto das creches de Campina Grande-PB $(11,2 \%)^{6}$ e em área de cobertura da ESF de Teresina-PI $(13,3 \%)^{14}$. Ainda, é similar à prevalência média ponderada $(11,3 \%)$ registrada para famílias que frequentam serviços públicos de saúde/favorecidos pelo PBF em revisão sistemática da literatura ${ }^{5}$.

Os dados para este estudo foram coletados no segundo semestre de 2014, que coincide com o início do processo de recessão da economia brasileira, a redução no consumo de bens e serviços e a diminuição de investimentos ${ }^{15}$. Além dos efeitos no emprego e nos rendimentos das famílias, a crise econômica tem produzido aumento da pobreza extrema e da desigualdade social, com demandas associadas a benefícios sociais relacionados à renda. Assim, os efeitos no consumo alimentar devem ser considerados na medida em que o critério de escolha dos alimentos na população de baixa renda é o preço ${ }^{16}$. Avigora-se, assim, uma situação de vulnerabilidade extrema em 15 de cada 100 domicílios da ESF, nos municípios estudados, nos quais há necessidade de reduzir a alimentação das crianças, que podem ficar o dia inteiro sem comer por falta de dinheiro ${ }^{10}$.
A associação encontrada neste estudo entre os processos diarreicos nas crianças e a insegurança alimentar e nutricional moderada/grave coincide com resultados de âmbito nacional ${ }^{17,18}$ e refletem a maior vulnerabilidade à diarreia em situação de insegurança alimentar e nutricional. Além disso, mostra a capacidade da EBIA de capturar riscos de insegurança alimentar e nutricional para problemas de saúde que estão em posição hierárquica superior aos fatores socioeconômicos ${ }^{17,18}$. Nesse sentido, as doenças diarreicas em crianças destacam-se, uma vez que caracterizam uma trajetória de sistematização com etiologia e ecologia variada e complexa, delimitada principalmente em contextos de pobreza, baixa escolaridade, condições de higiene precárias, acesso limitado aos serviços de saúde e situação alimentar e nutricional deficitária ${ }^{19}$.

Em inquérito populacional que compreendeu famílias das regiões Nordeste e Sul do Brasil, de forma similar aos resultados do atual estudo, maior probabilidade de insegurança alimentar e nutricional moderada/grave foi identificada em domicílios com mães de escolaridade inferior, menor renda familiar per capita e beneficiários do $\mathrm{PBF}^{1}$. Essas associações também foram confirmadas em estudos anteriores, incluindo diferentes países (Argentina, Angola, México) e contextos epidemiológicos no Brasil, com a insegurança alimentar e nutricional, inclusive os graus mais severos ${ }^{3,12-14,20-26}$. Altas prevalências de insegurança alimentar e nutricional entre beneficiários do PBF também têm sido constatadas ao estudar apenas famílias que usufruem do benefício ${ }^{24}$.

A baixa escolaridade materna pode dificultar a inserção no mercado de trabalho formal e contribuir para a ocorrência da insegurança alimentar e nutricional ${ }^{24}$. Além disso, pode ser um fator negativo relacionado ao acesso de informação apropriada e à escolha de uma alimentação de qualidade que pode prejudicar principalmente as crianças $^{12}$. É pertinente considerar que a alimentação adequada e saudável se refere à realização do direito ao acesso, permanente e regular, a uma pratica alimentar adequada aos aspectos biológicos e sociais dos indivíduos com base nos princípios de variedade, equilíbrio, moderação, prazer (sabor), dimensões de gênero e etnia, e formas de produção ambientalmente sustentáveis ${ }^{27}$. As equipes de saúde da ESF têm uma grande importância na promoção da segurança alimentar e nutricional nesse contexto.

A renda per capita exerce função essencial na compra de alimentos e, portanto, como determinante da insegurança alimentar e nutricional ${ }^{20,23}$. 
Essa relação é relevante, ainda, do ponto de vista metodológico, pois coloca a EBIA como medida viável na avaliação de iniquidades ${ }^{5}$.

Segundo os resultados apresentados, a maior vulnerabilidade das famílias beneficiárias do PBF aos graus mais intensos de insegurança alimentar e nutricional sugere uma correta focalização do Programa, mas também que o mesmo não está sendo capaz de garantir a segurança alimentar e nutricional dessas famílias. Isso remete à necessidade de aumentar os valores pagos pelo benefício, combinado a outras estratégias de aumento de escolaridade, emprego e renda ${ }^{1,9,12,13}$ que devem considerar, ainda, a conjuntura da atual crise econômica. Cabe destacar, também, a importância de analisar o efeito do tempo de permanência no PBF na insegurança alimentar e nutricional, o que ficou fora do alcance do presente estudo.

$\mathrm{Na}$ conjuntura analisada, é preciso enfatizar a necessidade de direcionar esforços para o enfrentamento de todas as formas de má nutrição associadas à insegurança alimentar e nutricional, possivelmente com conquistas estacionadas pela crise. Com essa meta, a Década de Ação em Nutrição (2016-2025) foi estabelecida como marco prioritário para as ações de nutrição, com foco principal no segundo objetivo do desenvolvimento sustentável que visa acabar com a fome, alcançar a segurança alimentar e nutricional, melhorar a nutrição e promover a agricultura sustentável. Como signatário da Década de Ação em Nutrição, o Brasil apresenta sua convenção em seis pilares, destacando em um deles a importância de os sistemas de saúde efetivamente incorporarem ações de nutrição que impactem nos problemas nutricionais ${ }^{28}$. É bom lembrar que o direito à alimentação adequada deve ser garantido por meio de políticas públicas de segurança alimentar e nutricional de responsabilidade do Estado e da sociedade ${ }^{29}$.

Considerando o local selecionado para o desenvolvimento desta pesquisa, é relevante reforçar que a ESF constitui espaço privilegiado para o desenvolvimento das ações de alimentação e nutrição, e qualificação da atenção à saúde na atenção primária ${ }^{30}$, tendo como eixos de assistência a promoção de ações integrativas e intersetoriais de educação em saúde e nutrição; a realização do cuidado nutricional em todas as fases do curso da vida; o desenvolvimento de planos terapêuticos para doenças crônicas; a intervenção nas deficiências nutricionais e o estímulo à produção e ao consumo de alimentos saudáveis ${ }^{31}$. Adicionalmente, a EBIA merece atenção por parte dos profissionais de saúde, pois representa um instrumento de fácil aplicação que pode ser usado para o diagnóstico da insegurança alimentar e nutricional como base do planejamento, da organização e da implementação de ações de cuidado nutricional $^{32}$.

$\mathrm{Na}$ tentativa de preservar a rotina do trabalho nos serviços de saúde, é possível a existência de viés na seleção da amostra do presente estudo. Adicionalmente, a observação de crianças condicionada aos atendimentos nas consultas de puericultura pode ter propiciado a inclusão de um grupo com condições mais precárias em termos de saúde. Entretanto, essas circunstâncias foram provavelmente minimizadas contemplando uma amostra aleatória a partir dos encaminhamentos realizados pelos agentes comunitários de saúde. Neste momento global em que a Nutrição é uma prioridade, os achados desse estudo podem servir de subsídio para um diagnóstico da situação de insegurança alimentar e nutricional dos municípios envolvidos. Adicionalmente, os indicadores, utilizados em conjunto com os das pesquisas desenvolvidas no município de Campina Grande, poderão servir de referência para o monitoramento da execução de ações públicas vinculadas ao Plano Estratégico de Desenvolvimento Paraíba 2040. Complementarmente, com base nos dados produzidos, é possível refletir sobre o papel das políticas públicas no combate histórico da insegurança alimentar e nutricional, e na garantia do direito humano à alimentação adequada.

\section{Conclusão}

A prevalência de insegurança alimentar e nutricional foi encontrada em seis a cada 10 famílias, predominando as formas moderada/grave, que foram mais prevalentes nos casos de vulnerabilidades na situação de saúde da criança e socioeconômica. Famílias das áreas de cobertura da ESF, sobretudo beneficiárias do PBF, representam um grupo vulnerável que, possivelmente, sofrem consequências da crise econômica brasileira, com implicações negativas nos graus mais severos de insegurança alimentar e nutricional. Fatores socioeconômicos tradicionais como as baixas renda e escolaridade continuam sendo variáveis associadas a essa problemática. Ações governamentais devem priorizar melhorias no PBF e nas condições de vida de forma a garantir o direito humano à alimentação e prevenir um possível aumento inoportuno da fome. 


\section{Financiamento}

Conselho Nacional de Desenvolvimento Científico e Tecnológico e Fundação de Apoio à Pesquisa do Estado da Paraíba.

\section{Aprovação ética}

Comitê de Ética em Pesquisa da Universidade Estadual da Paraíba.

\section{Referências}

1. Facchini LA, Nunes BP, Motta JVS, Tomasi E, Silva SM, Thumé E, Silveira DS, Dilélio AS, Saes MO, Miranda VIA, Volz PM, Osório A, Fassa AG. Insegurança alimentar no Nordeste e Sul do Brasil: magnitude, fatores associados e padrões de renda per capita para redução das iniquidades. Cad Saúde Pública 2014; 30(1):161-174.

2. Organización de las Naciones Unidas para la Alimentación y la Agricultura. Fondo Internacional de Desarrollo Agrícola. Organización Mundial de la Salud. Fondo de las Naciones Unidas para la Infancia. Programa Mundial de Alimentos. El estado de la seguridad alimentaria y la nutrición en el mundo 2017: Fomentando la resiliencia en aras de la paz y la seguridad alimentaria. Roma: FAO; 2017.

3. Poblacion AP, Marín-León L, Segall-Corrêa AM, Silveira JÁ, Taddei JAAC. Insegurança alimentar em domicílios brasileiros com crianças menores de cinco anos. Cad Saúde Pública 2014; 30(5):1067-1078.

4. Brasil. Lei no 11.346 , de 15 de setembro de 2006. Cria o Sistema Nacional de Segurança Alimentar e Nutricional - SISAN com vistas em assegurar o direito humano à alimentação adequada e dá outras providências. Diário Oficial da União 2006; 18 set.

5. Bezerra TA, Olinda RA, Pedraza DF. Insegurança alimentar no Brasil segundo diferentes cenários sociodemográficos. Cien Saude Colet 2017; 22(2):637-651.

6. Pedraza DF, Gama JSFA. Segurança alimentar e nutricional de famílias com crianças menores de cinco anos do município de Campina Grande, Paraíba. Rev Bras Epidem 2015; 18(4):906-917.

7. Bezerra TA, Figueroa Pedraza D. (In)segurança alimentar entre famílias com crianças menores de cinco anos residentes em área de vulnerabilidade social de Campina Grande, Paraíba. Rev Nutr 2015; 28(6):655665.

8. Motta LCS, Siqueira-Batista R. Estratégia Saúde da Família: Clínica e Crítica. Rev Bras Educ Med 2015; 39(2):196-207.

9. Cotta RMM, Machado JC. Programa Bolsa Família e segurança alimentar e nutricional no Brasil: revisão crítica da literatura. Rev Panam Salud Publica 2013; 3(1):54-60.

10. Segall-Corrêa AM, coordenadora. Acompanhamento e avaliação da segurança alimentar de famílias brasileiras: validação de metodologia e de instrumento de coleta de informação. Relatório Técnico: versão preliminar. Campinas: Unicamp; 2004.

11. Brasil. Instituto Brasileiro de Geografia e Estatística (IBGE). Pesquisa Nacional por Amostra de Domicílios: segurança alimentar, 2013. Rio de Janeiro: IBGE; 2014.

12. Ferreira HS, Souza MECA, Moura FA, Horta BL Prevalência e fatores associados à Insegurança Alimentar e Nutricional em famílias dos municípios do norte de Alagoas, Brasil, 2010. Cien Saude Colet 2014; 19(5):1533-1542.

13. Silva EKP, Medeiros DS, Martins PC, Sousa LA, Lima GP, Rêgo MAS, Silva TO, freire AS, Silva FM. Insegurança alimentar em comunidades rurais no Nordeste brasileiro: faz diferença ser quilombola? Cad Saúde Pública 2017; 33(4):e00005716. 
14. Sabóia RCB, Santos MM. Prevalência de insegurança alimentar e fatores associados em domicílios cobertos pela Estratégia Saúde da Família em Teresina, Piauí, 2012-2013. Epidemiol Serv Saúde 2015; 24(3):749-758.

15. Barbosa Filho FH. Crise econômica de 2014/2017. Estud Av 2017; 31(89):51-60.

16. Brasil. Instituto de Pesquisa Econômica Aplicada (IPEA). Políticas sociais: acompanhamento e análise. Brasília: IPEA; 2015.

17. Reis M. Food insecurity and the relationship between household income and children's health and nutrition in Brazil. Health Econ 2012; 21(4):405-427.

18. Gubert MB, Spaniol AM, Bortolini GA, Pérez-Escamilla R. Household food insecurity, nutritional status and morbidity in Brazilian children. Public Health Nutr 2016; 19(12):2240-2245.

19. Vasconcelos MJOB, Rissin A, Figueiroa JN, Lira PIC, Batista Filho M. Doenças diarreicas e hospitalizações em menores de cinco anos no estado de Pernambuco, Brasil, nos anos de 1997 e 2006. Cien Saude Colet 2018; 23(3):715-722.

20. Cabral CS, Lopes AG, Lopes JM, Vianna RPT. Segurança alimentar, renda e Programa Bolsa Família: estudo de coorte em municípios do interior da Paraíba, Brasil, 2005-2011. Cad Saúde Pública 2014; 30(2):393402.

21. Bittencourt LS, Santos SMCD, Pinto EDJ, Aliaga MA, Ribeiro-Silva RC. Factors associated with Food Insecurity in Households of Public School Students of Salvador City, Bahia, Brazil. J Health Popul Nutr 2013; 31(4):471-479.

22. Aquino JDS, Sequeira-de-Andrade LAS, Silva PEBAD, Silva APD, Vieira CRDS, Lira PICD. Food insecurity and socioeconomic, food and nutrition profile of schoolchildren living in urban and rural areas of Picos, Piauí. Rev Nutr 2014; 27(4):395-404.

23. Marin-Leon L, Francisco PMSB, Segall-Corrêa AM, Panigassi G. Bens de consumo e insegurança alimentar: diferenças de gênero, cor de pele autorreferida e condição socioeconômica. Rev Bras Epidemiol 2011; 14(3):398-410.

24. Sperandio N, Priore SE. Prevalência de insegurança alimentar domiciliar e fatores associados em famílias com pré-escolares, beneficiárias do Programa Bolsa Família em Viçosa, Minas Gerais, Brasil. Epidemiol Serv Saúde 2015; 24(4):739-748.
25. Esturk O, Oren MN. Impact of household socio-economic factors on food security: case of Adana. Pakistan J Nutr 2014; 13(1):1-6.

26. Mundo-Rosas V, Méndez-Gómez I, Shamah-Levy T. Caracterización de los hogares mexicanos en inseguridad alimentaria. Salud Pública Mex 2014; 56(1):S12-S20.

27. Conselho Nacional de Segurança Alimentar e Nutricional (CONSEA). Relatório Final III Conferência Nacional de SAN: por um desenvolvimento sustentável com soberania e segurança alimentar e nutricional. Fortaleza: CONSEA; 2007.

28. Câmara Interministerial de Segurança Alimentar e Nutricional (CAISAN). Compromissos do Brasil para a Década de Ação das Nações Unidas para a nutrição (2016-2025). Brasília: CAISAN; 2015.

29. Maluf RSJ. Direito humano à alimentação adequada e saudável. In: Maluf RSJ. Segurança alimentar e nutricional. $3^{\text {a }}$ ed. Petrópolis: Vozes; 2011. p. 20-21.

30. Pimentel VRM, Sousa MF, Hamann EM, Mendonça AVM. Alimentação e nutrição na Estratégia Saúde da Família em cinco municípios brasileiros. Cien Saude Colet 2014; 19(1):49-58.

31. Borelli M, Domene SMA, Mais LA, Pavan J, Taddei JAAC. A inserção do nutricionista na Atenção Básica: uma proposta para o matriciamento da atenção nutricional. Cien Saude Colet 2015; 20(9):2765-2778.

32. Peixoto MRG, Ramos K, Martins KA, Schincaglia RM, Braudes-Silva LA. Insegurança alimentar na área de abrangência do Núcleo de Apoio à Saúde da Família em Itumbiara, Goiás. Epidemiol Serv Saúde 2014; 23(2):327-336.

Artigo apresentado em 20/06/2018

Aprovado em 05/06/2019

Versão final apresentada em 07/06/2019

Editores-chefes: Romeu Gomes, Antônio Augusto Moura da Silva 